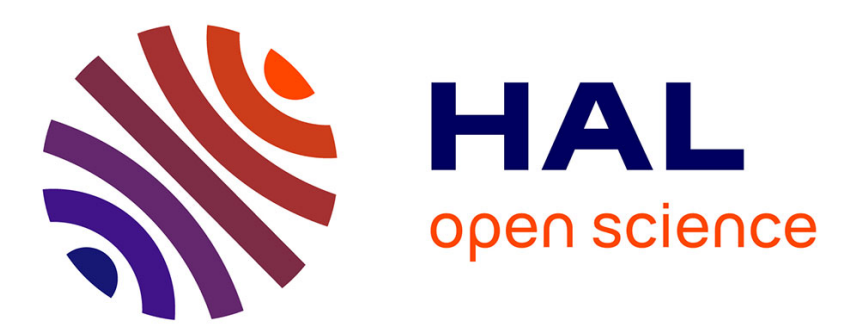

\title{
2DVD Data Revisited: Multifractal Insights into Cuts of the Spatiotemporal Rainfall Process
}

\author{
Auguste Gires, Ioulia Tchiguirinskaia, D Schertzer, A. Berne
}

\section{To cite this version:}

Auguste Gires, Ioulia Tchiguirinskaia, D Schertzer, A. Berne. 2DVD Data Revisited: Multifractal Insights into Cuts of the Spatiotemporal Rainfall Process. Journal of Hydrometeorology, 2015, 16 (2), pp.548-562. 10.1175/jhm-d-14-0127.1 . hal-01238345

\section{HAL Id: hal-01238345 \\ https://hal-enpc.archives-ouvertes.fr/hal-01238345}

Submitted on 12 Feb 2018

HAL is a multi-disciplinary open access archive for the deposit and dissemination of scientific research documents, whether they are published or not. The documents may come from teaching and research institutions in France or abroad, or from public or private research centers.
L'archive ouverte pluridisciplinaire HAL, est destinée au dépôt et à la diffusion de documents scientifiques de niveau recherche, publiés ou non, émanant des établissements d'enseignement et de recherche français ou étrangers, des laboratoires publics ou privés. 
1Title: 2DVD data revisited: multifractal insights into cuts of the spatio-temporal rainfall 2process.

3

4Authors:

5Auguste Gires ${ }^{(1)^{*}}$, Ioulia Tchiguirinskaia ${ }^{(1)}$, Daniel Schertzer ${ }^{(1)}$, Alexis Berne $^{(2)}$

6(1) U. Paris-Est, Ecole des Ponts ParisTech, LEESU, Marne-la-Vallée, France

7(2) Ecole Polytechnique Fédérale de Lausanne, Laboratoire de Télédétection

8Environnementale, Lausanne, Switzerland

9* Corresponding author : auguste.gires@leesu.enpc.fr; +33 164153648 ; Ecole des Ponts

10ParisTech, LEESU, 6-8 avenue Blaise Pascal, Cité Descartes, Champs sur Marne, 77455

11Marne-La-Vallée Cedex 2

12

13Abstract

14 Data collected during 4 heavy rainfall events that occurred in Ardèche (France) with 15the help of a 2D Video-disdrometer are used to investigate the structure of the rain drops 16distribution in both space and time. A first type of analysis is based on the reconstruction of 1736-m height vertical rainfall columns above the measuring device. This reconstruction is 18obtained with the help of a ballistic hypothesis applied to $1 \mathrm{~ms}$ time step series. The 19corresponding snapshots are analysed with the help of Universal Multifractals. For 20comparison, a similar analysis is performed on the time series for $1 \mathrm{~ms}$ time step, as well as 21time series of accumulation maps of $\mathrm{N}$ consecutive recorded drops (therefore with variable 22time steps).

23 It turns out that the drop distribution exhibits a good scaling behaviour on the range 240.5 - 36 m during the heaviest portion of the events, confirming the lack of empirical 25evidence of the widely used homogenous assumption for drop distribution. For smaller scales 
26drop positions seems to be homogeneously distributed. The notion of multifractal singularity 27is well illustrated by the very high resolution time series.

28

29

\section{1) Introduction}

31

32 Rainfall is extremely variable over wide range of scales both in space and time. It has 33become rather usual to characterize this behaviour with the help of scaling properties, and 34more recently multifractals (see Lovejoy and Schertzer 1995, Schertzer et al. 2010 for 35reviews). This framework is physically based in the sense that it is more than a simple tailored 36statistical framework. Indeed it relies on the cascade process concept which was introduced to 37reflect the scale invariance properties of the Navier-Stokes equations that govern atmospheric 38dynamics. It is assumed that the unknown equations governing rainfall inherit these properties 39of scale invariance (Schertzer and Lovejoy 1987, Hubert et al. 1993). Since the advent of this 40framework in the 1980s, ample empirical evidence have established its relevancy on scales 41ranging from few minutes to decades in time and hundreds of meters to planetary size in 42space (Lovejoy et al. 2008). Some authors reported not a single scaling regime but various 43ones separated by breaks; typically at few minutes and few days in time and few kilometres in 44space. Various types of data have been used; rain gauges (de Lima and de Lima 2009, de Lima 45and Grasman 1999, Fraedrich and Larnder 1993, Ladoy et al. 1993, Olsson 1995, Tessier et al. 461996), disdrometers (de Montera et al. 2009, Gires et al. 2014), weather radars (Gires et al. 472011 2012, Nykanen and Harris 2008, Tessier et al. 1993, Verrier et al. 2010), satellite with 48TRMM data (Lovejoy et al. 2008) and even numerical outputs of climate simulations (Royer 49et al. 2008) or meso-scale models (Gires et al. 2011). 
51scale down to which atmospheric turbulence - the embedding field of rainfall - is known to 52exhibit scaling behaviour (see Anselmet et al. 2001 for a review). Hence the need to 53investigate more precisely the minimum spatio-temporal scales down to which scaling is 54observed in rainfall fields. Very few studies have analysed this, mainly because of the lack of 55rainfall data at these scales. Mandapaka et al. (2009) observed on lidar data scaling on the 56range 1 - 512 s in time and 2.5 - 320 m in space. Lilley et al. (2006) and Lovejoy and 57Schertzer (2008), using few 3D snapshots of an $8 \mathrm{~m}^{3}$ volume with most of its drops 58(Desaulnier-Soucy et al. 2001), reported a scaling behaviour down to few tens of cm with a 59dependency on the turbulence intensity and drop size. Lovejoy and Schertzer (1990) or 60Gabbella et al. (2001) using large ( m) sheets of chemically blotted paper also found a 61scaling behaviour down to almost drop scale but these results have been disputed (Jameson 62and Kostinski 1998). In this paper we suggest to investigate more in depth the spatio-temporal 63(3 dimensions in space and 1 dimension in time) features of rainfall fields down to drop scale 64using data collected by a 2D video-disdrometer denoted 2DVD hereafter (see Kruger and 65Krajewski 2002, for a precise description of the device's functioning), deployed in the 66Ardèche region (South-East of France) in the framework of the HyMeX campaign (Ducrocq 67et al. 2014), in an innovative way. Indeed this device has been extensively used as a reference 68in comparison with other rainfall measuring ones (Krajewski et al. 2006, Tokay et al. 2013), 69or to investigate drops and more generally hydrometeor shape (Battaglia et al. 2010, Cao et al. 702008, Thurai and Bringi 2005) and size (Thurai et al. 2011) distribution features, but not to 71address this issue of the spatio-temporal structure of rainfall process at drop scale. More 72precisely vertical, horizontal and temporal cuts of rainfall fields are either obtained or 73reconstructed with the data provided by a 2DVD and investigated with the help of multifractal 74techniques. 
A related issue is whether drops are homogeneously distributed in space and time.

76Scaling laws are incompatible with a homogenous distribution (Poisson statistics), but it 77remains a debated topic. It has mainly been discussed in time by analysing drop counts over 78various time steps. Most authors have reported deviations from Poisson statistics also not 79necessarily attributing them to an underlying scaling behaviour; Kostinski and Jameson 80(1997) used 1 min drop counts by Joss-Waldvogel disdrometer (1967) to most likely 81invalidate a Poisson framework during heavy rainfall periods (more than 27 drops / min), and 82confirmed this with $1 \mathrm{~s}$ (corresponding to few meters) counts computed with 15 min of 2DVD 83data (Jameson and Kostinski 1999), noticing that larger drops are more correlated over longer 84coherence time. Uijlenhoet et al. (1999) also observed deviations from Poisson statistics with 8535 min of 10 s time step disdrometer data ( 6500 drops), but found that the discrepancies are 86only due to small drops (with diameter smaller than $1.1 \mathrm{~mm}$ ) which basically do not influence 87rain rates or reflectivity which are related to higher order moments of the drop size 88distribution. At the inter-event scale (4 months of data) Lavergnat and Golé (1998) observed a 89power law decrease of the duration between the observations of two consecutive drops with a 90disdrometer, which is incompatible with Poisson statistics. Hence in this paper we suggest to 91also tackle this issue by systematically comparing (when possible!) our results with those that 92would have been obtained with homogeneously distributed drops. Such methodology was 93already employed in Lilley et al. (2006) and Jameson and Kostinski (1998).

94 The collected data is presented in section 2 along with the basic ideas underlying the 95multifractal framework. Section 3 describes the analysis of snapshots of reconstructed $36 \mathrm{~m}$ 96high vertical columns of air above the device with all its drops. The high temporal resolution 97of the measuring device is used in section 4 to study $1 \mathrm{~ms}$ time step series (over the $11 \mathrm{x} 11 \mathrm{~cm}^{2}$ 98sampling area of the device). Drop accumulation maps (therefore with varying time steps, but 99for the same sampling area) are analysed in section 5. 


\section{2) Data description and multifracal framework}

102

1032.1) Data description

104 The data used in this paper were collected with the help of a 2DVD (Kruger and 105Krajewski 2002). The 2DVD provides detailed information about the geometry and the fall 106velocity of the particles falling through its sampling area of about $11 \times 11 \mathrm{~cm}^{2}$, by means of two 107perpendicular high-speed line cameras (with a pixel size of about $0.2 \mathrm{~mm}$ and a time 108resolution of about 1ms). From the two reconstructed side views of each raindrop, the shape 109and equivolume diameter are retrieved (assuming the rain drops are oblate spheroids). The 110two cameras being shifted in the vertical by about $6.5 \mathrm{~mm}$, the fall velocity of each particle is 111directly measured. The same raw data will be used to generate three types of representation 112and analysis in the following sections: vertical reconstructed columns, high resolution time 113series and small scale accumulation maps. Methods used to obtain each cut of the underlying 114spatio-temporal rainfall representation are included in separated, dedicated section to facilitate 115the reading of the paper.

116 The device was installed in Le Pradel, Ardèche, France (see Fig. 1) during the fall 1172012 and 2013 in the framework of the HyMeX project (Ducroq et al., 2014). The considered 1182DVD is the low version (with reduced wind disturbance). A number of rainfall events were 119collected, and the two heaviest ones (in terms of 5-min rain rate) for each fall have been 120selected for the present study. Their main features are summarized in Tab. 1.

\section{2) Multifractal framework}

123 The basic principle of the multifractal framework is briefly reminded. For more details 124refer to the recent review by Scherter and Lovejoy (2011). Multifractals are used to 
125characterize and also simulate geophysical fields extremely variable over a wide range of 126spatio-temporal scales which also exhibit long range correlation. It basically relies on the 127concept of multiplicative cascades. Let us denote $\varepsilon_{\lambda}$ such a field at resolution $\lambda(=L / l)$ 128defined as the ratio between the outer scale $L$ of the phenomenon and the observation scale $l$. 129The field at a given resolution $\lambda$ is obtained from the field at the maximum resolution $\Lambda$ by 130averaging it over pixels of resolution $\lambda$.

131 Let us first introduce in a rather intuitive (but less rigorous mathematically) way the 132notion of multifractal fields with the help of the concept of fractal dimension. $A$ is a 133geometrical set embedded in a space of dimension $d$ (for instance $d=1$ for time series and $d=$ 1342 for maps) and $N_{\lambda}$ the number of non overlapping $d$-dimension boxes of size $l$ needed to 135cover A at resolution $\lambda(=L / l$, where $L$ is the outer scale). If the set is fractal then we have: $136 N_{\lambda} \approx \lambda^{D_{F}}$

137 where $D_{\mathrm{F}}$ is the fractal dimension of $A$.

138 The geometrical set corresponding to the portion of a field $\varepsilon_{\lambda}$ above a given threshold 139exhibits fractal features that can be quantified with the help of a fractal dimension. The 140decreasing dependency of this fractal dimension with regards to the considered threshold 141reflects the need of multiple fractal dimensions to characterize the field. In a more rigorous 142mathematical way the threshold is replaced by the scale invariant notion of singularity but the 143underlying idea remains the same.

145 More precisely if a field is scaling then its power spectra $E$ is a power-law with respect 146to the wave number $k$ :

$147 E(k) \approx k^{-\beta}$

148Where $\beta$ is the spectral slope. A larger $\beta$ reflects a weaker correlation. 
150field. In the multifractal framework all the moments are used to fully characterize the 151variability across scale. The statistical moment of order $q$ at a given resolution scales with the 152resolution:

$153<\varepsilon_{\lambda}{ }^{q}>\approx \lambda^{K(q)}$

154Where $K(q)$ is the moment scaling function. In the specific framework of Universal 155Multifractals (UM), which correspond to the stable and attractive limits of nonlinearly 156interacting multifractal processes (i.e. a multiplicative generalization of the central limit 157theorem), $K(q)$ has the following analytical expression :

$158 K(q)=\frac{C_{1}}{\alpha-1}\left(q^{\alpha}-q\right)+H q$

159which only depends on three parameters having a strong physical meaning (Schertzer and 160Lovejoy 1987 1997):

161- $H$, the degree of non-conservation, which measures the scale dependency of the mean 162field $. H=0$ for a conservative field, and can be either positive or negative corresponding to a 163fractional integration or differentiation respectively of a conserved field;

164- $C_{1}$, the mean intermittency co-dimension, which measures the mean sparseness of the field, 165i.e. how concentrated is the mean field. A homogeneous field fills the embedding space and 166has $C_{1}=0,0 \leq C_{1} \leq d$ where $d$ is the dimension of the embedding space (a greater $C_{1}$ could 167theoretically exit but it would correspond to fields almost surely null everywhere)

168- $\alpha(0 \leq \alpha \leq 2)$, the multifractality index, which measures the variability of the intermittency, 169i.e. its dependence with respect to the considered level of activity. When $\alpha=0$, it means that all 170activity levels exhibit the same intermittency reflecting a fractal field. 
173The various parameters are related by the following equation from which $H$ is usually 174estimated:

$175 \beta=1+2 H-K(2)$

176

177

1783) Analysis of reconstructed vertical rainfall columns

179

1803.1) Ballistic reconstruction of a column

181

182 As already explained, the 2DVD provides for each drop a direct measurement of the $183 f a l l$ velocity and of the horizontal position within the $11 \mathrm{x} 11 \mathrm{~cm}^{2}$ sampling area of the device. 184Assuming the validity of the hypothesis of vertical ballistic trajectories, i.e. that both remain 185constant during the last seconds of fall and equal to the ones measured near ground surface, it 186is possible to reconstruct the trajectory of each drop. Achieving this for all the drops enables 187to reconstruct the whole rainfall field drop by drop on a column above the device (Fig 2.a). 188The height of the column studied in this paper is $36 \mathrm{~m}$.

189 The ballistic assumption is extremely coarse. Indeed by assuming an underlying 190laminar flow, we neglect all interactions with the turbulent wind field and notably shear 191effects, which have an influence on drops velocity, especially for small ones. Neglecting these 192effects tend to worsen the quality of the scaling we can observe with the help of this 193reconstruction. Drop population dynamics (e.g. coalescence in case of collisions, break-ups) 194is also ignored although wind tunnels studies have shown that raindrop interactions can occur 195on scales below 36 m (Cotton and Gokhale 1967). Possible effects on the small drop tendency 196to collide or not, due to small scale turbulence self induced by the aerodynamic forces exerted 197on the larger falling raindrop (for large raindrops the Reynolds number is of the order of few 
198thousands) is also not taken into account. However, despite its limitations, this reconstruction 199can yield some preliminary insight before considering more complex reconstruction, e.g. with 200randomized trajectories taking into account turbulence effects as well as coalescence and 201breakup

202 Finally, the column is divided into 8192 boxes of $4.3 \mathrm{~mm}$ height, the two horizontal 203dimensions being the horizontal sampling area ones’ i.e. $11 \mathrm{~cm}$ (Fig. 2.b). Although the 204minimum inter-drop distance (defined as the drop concentration to the power -1/3) observed 205for the studied events is roughly $4 \mathrm{~cm}$ (and usually much larger), the height of the boxes is 206chosen much smaller so that results can be compared in the vertical and in the horizontal 207dimension (see section 5). For each box we consider the sum of the $p$-th power of the volumes $208 V_{i}$ of the drops (computed with help of the equivolume diameter $D_{i}$ estimated by the 2DVD 209device) contained in this box:

$210 \chi_{p}=\sum V_{i}^{p}$

211As suggested by Lilley et al (2006) by varying $p$ various physical quantities are represented;

212 for instance $\chi_{\mathrm{p}}$ for $p$ equal to $0,1,7 / 6$ and 2 is respectively proportional to the drop 213concentration, Liquid Water Content (LWC), rain rate (assuming a fall velocity proportional 214to the square root of the drop diameter), and radar reflectivity (which is nearly proportional to 215the 6-th power of the diameter; in the Rayleigh scattering regime). This yields a spatial 1D 216(vertical) field. An example of vertical evolution of the LWC (basically $\chi_{\mathrm{p}}$ for $p=1$ ) within the 217column during the heaviest portion of the 09-24-2012 event is displayed in Fig 2.c. Such 218reconstructed snapshot of the vertical column above the 2DVD is done every second. Before 219going on, it should be mentioned that small drops are not fully represented with the 2DVD 220since measurements for drops with an equivolume diameter smaller than $0.3 \mathrm{~mm}$ are 221unreliable (Tokay et al 2013). It means that small moments are likely to be biased. 222Nevertheless, the quantities of interest studied in this paper are for $\mathrm{p}>1$ (moment of the 
223diameter greater than 3) meaning that this limitation does not have a strong influence on the 224discussed results.

225 In order to compare the observed properties of the fields with the ones that would be 226obtained if drops were homogeneously distributed, pseudo-synthetic fields are also generated. 227To obtain a realization for a given column, the drop centres are re-assigned with the help of a 228random, uniform distribution, whereas the drop sizes are unchanged. The vertical evolution of 229the LWC obtained for a realisation is displayed Fig. 2.c.

2323.2) Scaling behaviour

233

234 Statistical scaling properties are obtained by ensemble averaging (each field sample is 235first independently upscaled, i.e. its resolution is degraded by averaging over adjacent pixels, 236then raised to various power $q$, and finally the ensemble average is performed to obtain an 237estimate of the theoretical moments and its scaling behaviour -Eq. 3-) over 60 consecutive 238column snapshots (1 min in physical time). The spectral analysis of the field (Eq. 2 in log-log 239plot) is displayed in Fig. 3.a for the 60 time steps starting on 24 September 2012 at 02:17 240UTC with $p=1$ (i.e. LWC estimate). This is a very intense period of the storm where there 241are 5573 drops in the column on average (which corresponds to roughly 13000 drops per $\mathrm{m}^{3}$ ) 242and the rain rate is approximately $180 \mathrm{~mm} \cdot \mathrm{h}^{-1}$. The rain rate is computed as the average rain 243rate measured at the level of the 2DVD device (bottom of the column). It is only an indication 244of the current rainfall intensity since some of the drops present in the studied 60 consecutive 245snapshots do not reach the device during this minute, meaning that consistent comparison is 246not possible. We mention it simply because rain rates are much more often used in 247hydrometeorology than drop concentrations. 
249(log $\mathrm{k} / \mathrm{k}_{0} \sim 3$ with $\left.\mathrm{k}_{0}=1 \mathrm{hz}\right)$. For smaller scales the spectra is flat as it would be for a white noise 250resulting from randomly homogeneously distributed drops. These findings are confirmed by 251the Trace Moment (TM) analysis (Eq. 3 in log-log plot) displayed Fig. 3.b for the whole range 252of available scales and on Fig. 3.c with a zoom on larger scales. It appears that the 253reconstructed fields exhibit a scaling behaviour (the coefficients of determination $r^{2}$ of the 254linear portions are all greater than 0.99) up to approximately $\lambda=64$ which corresponds 255roughly to $0.5 \mathrm{~m}$, a value rather similar to the one found in the spectral analysis. This 256confirms, on an extended range of scales and using more data, the results of Lilley et al. 257(2006) who observed scaling from $0.4 \mathrm{~m}$ to $2 \mathrm{~m}$ on few snapshots of $8 \mathrm{~m}^{3}$ volume with most 258of its drops. This suggests that drops are distributed in a scaling manner down to $0.5-1 \mathrm{~m}$ and 259below they are homogeneously distributed, which is in agreement with the findings of 260Lovejoy and Schertzer (2008).

261 Similar analyses are also performed on the synthetic fields with homogeneously 262distributed drop positions. The spectra remains flat on the whole range of scales (Fig 3.d) and 263the TM analysis curves (solid lines on Fig. 3.b - 3.c) do not exhibit any scaling regime (i.e. 264linear portion). More quantitatively, over the scaling regime identified for reconstructed 265fields, the $r^{2}$ for the homogeneous fields are typically equal to 0.80 . The curves for the 266smallest scales (roughly $4 \mathrm{~mm}$ - few $\mathrm{cm}$ ) are very similar between reconstructed and 267homogeneous fields which confirms the results of the spectral analysis for this range of 268scales. For larger scales, deviations between reconstructed and homogeneous fields are visible 269with a transition regime before the scaling regime of the reconstructed fields (0.5-36 m). Very 270similar results are found for other realisations of homogeneously distributed fields showing 271that these results are statistically meaningful, i.e the Poisson hypothesis of a homogeneous 
272drop distribution, which is commonly used to model rainfall, is not correct for this minute of 273the storm. Indeed it does not enable to reproduce the observed structure of drop distributions. An analysis of the other minutes of this storm and the others lead to qualify this

275statement. Indeed it appears that a scaling behaviour is retrieved only when there are enough 276drops in the column (typically more than 2000). To illustrate this, Fig. 4.a displays TM 277analysis for 60 s starting at 02:23 UTC for the same 09-24-2012 event (415 drops on average 278in the column, approximately $\left.30 \mathrm{~mm} \cdot \mathrm{h}^{-1}\right)$. In this case with less numerous drops the linear 279portions on the TM curves tend to disappear and furthermore the spectra for large scales 280flattens with $\beta$ estimates closer to zero, suggesting a behaviour more in agreement with a 281homogeneous distribution of drops. To get an idea of the number of drops needed in the 282vertical columns to observe scaling Fig 4.b displays a scatter plot of the $r^{2}$ of the TM curve for $283 q=1.5$, which is taken as an indication of the quality of the scaling, vs. the average number of 284drops in the studied vertical columns for the 09-24-2012 event (60 consecutive snapshots are 285used for each point). Similar plots are obtained for the other events. It appears that scaling is 286visible only when there are more than 2000-3000 drops in the column, which is the case for 287roughly 500 snapshots (8 min) during the studied events. In order to get a similar threshold 288with rain rates, Fig. 4.c displays the rain rate computed vs. the average number of drops in the 289studied vertical columns for the same minute. The limitations of the comparison between 290these two quantities are visible on this figure especially for extreme minutes for which the 291relation is not linear. The indicative rain rate threshold to observe scaling in the vertical 292columns would be of roughly $75 \mathrm{~mm} \cdot \mathrm{h}^{-1}$.

293 For the vertical columns exhibiting a scaling behaviour, we find for scales ranging 294from $35 \mathrm{~m}$ to $0.5 \mathrm{~m} \alpha \sim 1.8-2, C_{1} \sim 0.005-0.01$ and $\beta \sim 1$ - 1.4 (slightly smaller than the values 295found by Lovejoy and Schertzer, 2008). With the help of Eq. 5 it leads to $H \sim 0-0.2$. $H$ is 296small enough so that it is relevant to directly implement the TM analysis on the vertical series 
297and not on their fluctuations. It is timely to mention that only a limited portion of the scaling 298regime is observed with this data set since there is only a ratio of 64 between the maximum 299and the minimum resolution of the scaling regime. It means that the reliability of the 300multifractal exponent estimates is not very high and their values should not be over301interpreted. We will therefore only briefly discuss them. The UM parameter estimates for the 302actual field are slightly different from the ones found by Lilley et al. (2006), with greater $\alpha$ 303and smaller $C_{1}$, and the storm to storm variability is also less pronounced but it could be due 304to the fact that here the estimates are discussed only for the most intense portion of the events, 305wheras Lilley et al. performed their analysis only on a limited number of volume snapshots 306from various storms and not necessarily obtained during the peak in rain intensity.

307 The UM parameter estimates are slightly different with especially a $C_{1}$ smaller than the 308ones usually reported at coarser resolution $\left(\alpha \sim 1.7-1.9, C_{1} \sim 0.05-0.2\right)$ by authors who studied 309rainfall field in space (Mandapaka et al. 2009, Verrier et al. 2010, Gires et al. 2013). Although 310these authors studied rainfall fields horizontally which makes direct comparison harder (due 311to a possible anisotropy between the vertical and horizontal directions), this hints at a possible 312break between two scaling regimes, a small scales one and a large scales one, located at few 313tens of meters or few hundreds of meters. Very high resolution data on larger areas would be 314needed to confirm this.

315 The UM estimates are also different from those found for wind turbulence with again 316greater values of $\alpha$ and smaller values of $C_{1}$ (Lazarev et al. 1994, Fitton et al. 2011). It would 317mean that there is no trivial link between the drop distributions and the wind turbulence field 318in which they are embedded and from which they presumably inherit the scaling behaviour up 319to a given (high) degree, contrary to the ballistic assumption that was used for the column 320reconstruction and could partially explain the observed differences with wind turbulence 
321parameters. Development of new instruments providing the size and 3D velocity of each rain 322drop over few tens of $\mathrm{m}^{3}$ would be required to properly address this issue.

323 With the UM parameter estimates found, one can expect sampling limitation and 324divergence of moment (see Schertzer and Lovejoy 2011 for more details) to affect estimates 325of $K(q)$ only for $q$ greater than 10, meaning that for lower statistical order the observed 326scaling is not spurious.

327 The same analyses were performed with other values of $p$ or only considering drops 328with an equivolumic diameter $D$ belonging to a given interval. Taking a greater $p$ or only 329larger drops yields similar results as expected since greater moments enhance the influence of 330large drops in Eq. 6. There is no significant difference in the scaling for drops up to $2.5 \mathrm{~mm}$ 331(the average number of drops per column remains significant) or $p$ smaller than 3 . A tendency 332of $\alpha$ to decrease and $C_{1}$ to increase with larger drops is noticed. For drops with $D>2.5 \mathrm{~mm}$ or $333 p>3$, the scaling is lost and the discrepancies with the Poisson framework are much less 334pronounced. An explanation (Lilley et al. 2006) is that large drops decouple from atmospheric 335turbulence, from which they inherit the scaling behaviour, at a larger scale than small drops. It 336means that, although the ballistic assumption on the studied scales is likely to be more valid 337 for larger drops, on the limited range of available scales (the maximum range in $35 \mathrm{~m}$ ) it will 338not be possible to observe scaling behaviour. Another limitation to the study of large drops 339with this data set is the very low number of drops in the reconstructed columns (typically less 340than 80 for the period with the heaviest rainfall available) which leads to less reliable 341statistics. 
3464.1) Description of the data

348 The data provided by the 2DVD enables to compute a rain rate with time steps $\Delta t$ of 3491 ms, given by:

$350 R_{1 m s}=\frac{\sum_{i} V_{i}}{S \Delta t}$

351Where the sum over the volumes $V_{\mathrm{i}}$ of the drops is now preformed over all the drops that 352passed through the sampling area $S$ during $\Delta t$ (1 ms here). Note that we consider a constant 353sampling area (the maximum one) and do not take into account refinements with regards to 354edge effects, which can be assumed of second order. This very high resolution rain rate was 355computed for $35 \mathrm{~min}$ ( $2^{21}$ time steps) of the 09-24-2012 event and for $140 \mathrm{~min}$ ( $2^{23}$ time steps) 356of the other events. The portion with the greatest cumulative depth was selected for each 357event. Before going on, we should mention that authors are not advocating for the use of 1 ms 358time step series for routine hydrological applications. Unfortunately, these series are likely to 359suffer from strong sampling errors, see Frasson et al. 2011 or Jaffrain and Berne 2011 for an 360illustration of this issue with standard occlusion optical disdrometers having much smaller 361sampling area. Here we use this series to demonstrate that the mulifractal notion of 362singularity, mostly perceived at small scales, has also consequences at large scales. 363 The 1 ms rain rate time series is plotted Fig. 5.b for the 09-24-2012 event along with 364the same series with $1 \mathrm{~s}$ time steps (Fig. 5.c) and the more classical 1 min time step (Fig. 5.d) 365(i.e. as the average over 60000 consecutive time steps). The temporal evolutions of the 366number of drops recorded (Fig. 5.a) and of the average mass weighed diameter (Fig. 5.e) per $367 \mathrm{~ms}$ is also displayed. Note that due to the high temporal resolution, the "average" is in fact 368most of the time steps performed over a unique drop. Two striking features should be noted: 369there are $96 \%$ of zeros while the series was taken from what is commonly considered as a 
370extreme rainfall event (this percentage ranges from 97 to $99 \%$ for the other events), and the 371 maximum rain rate (over $1 \mathrm{~ms}$ ) is of $60000 \mathrm{~mm} / \mathrm{h}$ which is a value much greater than what 372hydro-meteorologists are used to (this maximum ranges from 30000 to $60000 \mathrm{~mm} / \mathrm{h}$ for the 373other events). These extremely high rain rates are due to the passing of several drops during 374the same ms; up to 14 during the 24 September event. Obviously, the diameter of the drops is 375also important, as it can be seen for the second peak (between minutes 7 and 10) of the event 376in Fig. 5.c which is not visible on the drop counts time series. From Fig. 5.e it appears that 377this second peak it is due to larger drops during this period. More standard values of rain rate 378are retrieved when averaging to the 1 min time steps.

379 These two features illustrate both the intermittency of the rainfall field and the notion 380of multifractal singularity, i.e. the fact that the rain rate is not point wise defined because it 381depends on the time interval over which it is estimated: it usually diverges when considering 382it for smaller and smaller intervals. Mathematically, it means that the rain accumulation is a 383singular measure with respect to the usual measure of time (i.e. the one dimensional Lebesgue 384measure). The need to properly deal with these two complex properties lead to the 385development of the theoretical framework of multifractals (see Schertzer and Lovejoy 2011 386for more details). In this framework the rain rate is expected to behave as: $387 R \approx \lambda^{\gamma}$

388When positive, the singularity $\gamma$ is the (algebraic) rate of divergence of the rain rate with the 389resolution. Here the singularity $\gamma$ corresponding to the maximum peak occurring at about 5 390min (Fig. 5) is equal to 0.8, 0.8 and 1.5 for time steps of $1 \mathrm{~ms}\left(\lambda=2.110^{6}\right), 1 \mathrm{~s}\left(\lambda=2.110^{3}\right)$ and $3911 \min (\lambda=35)$ respectively. The differences are due to the fact that there are several scaling 392regimes over these scales as will be shown in the next section. In order to emphasize this 393point on the whole time series, they are also displayed in Fig. 5 in a $\log _{\lambda}$ scale. 
3954.2) Temporal multifractal analysis

396

397 Figure 6 displays the spectral analysis and the TM analysis for the 10-23-2013 event 398for which the total duration taken into account is 140 min enabling to study a $2^{23}$ length time 399series (of 1 ms rain rates)! Such duration was chosen because it is the longest one enabling to 400remain in the event and to have a series whose length is a power of 2 as it is needed for the 401simplest TM analysis. Similar curves are obtained for the other events. The spectra does not 402reflect a very good scaling behaviour and it seems that there is a scaling breakdown for the 403frequency $k$ roughly equal to $70\left(\log \left(\mathrm{k} / \mathrm{k}_{0}\right) \sim 4.2\right.$ with $\left.\mathrm{k}_{0}=1 \mathrm{hz}\right)$, which corresponds to $2 \mathrm{~min}^{-1}$ 404(the breakdown is also at roughly $2 \mathrm{~min}^{-1}$ for the $09-24-2012$ event, the series of which is 405shorter). The coefficient of determination of the linear portion for large scales is weak (equal 406to 0.68 ), hence the estimates of the spectral slope $\beta$ are not reliable and will therefore not be 407discussed. No spectral slope can be derived for smaller scales. On the TM analysis three 408scaling regimes can be indentified: $140 \mathrm{~min}$ - few min (from 2 to 8 min according the event) 409with a rather good scaling $\left(r^{2}=0.97-0.9\right)$-blue lines in Fig. 6.b- ; a transition regime in the 410range few min - 32 ms with a rather bad scaling -red lines in Fig. 6.b-; and a very small scales 411regime $32 \mathrm{~ms}-1 \mathrm{~ms}$ with a good scaling $\left(r^{2}=0.99\right)$-green lines in Fig. 6.b-. The poor quality 412of the scaling observed on the spectral analysis means that the results of the TM analysis 413should not be "over-interpreted". We observe similar scaling regimes to those reported and 414discussed by Schertzer et al. (2012), i.e. the multifractal regime for 1 day - 7 min and the 415fractal regime for $2 \mathrm{~s}-1 \mathrm{~ms}$, obtained with the help of a multifractal analysis by 416Tchiguirinskaia et al. (2003) of an infrared optical spectro-pluviometer time series (Salles et 417al. 1998).

418 For the small scales (32 ms $-1 \mathrm{~ms}$ ) we indeed find an index of multifractality $\alpha$ 419roughly equal to zero meaning that the observed behaviour is monofractal. $C_{1}$ is in the interval 
$4200.7-0.85$ and estimates are roughly equal to the fractal co-dimension of the time series which 421means that this regime simply reflects the passing of individual drops through the sampling 422area. Given the poor quality of the scaling, UM estimates are not computed for the medium 423scales corresponding to the transition regime. With regards to large scales, the UM parameters 424estimates for all the events are reported in Table 2. The values of $\alpha$ are no longer equal to zero 425meaning that an actual multifractal behaviour coming from the common influence of several 426drops is retrieved (contrary to what was observed for small scales). The strong variations 427according to the event are much greater, especially for $\alpha$, than those expected if various 428realizations of the same process were analysed. It means that the UM parameters seem to 429depend on the event. The standard drop size distribution (DSD, $N(D)$ ) was computed for each 430event on average (not shown here) and it appears that the observed differences in the UM 431parameters are not related to DSD differences (especially to the thickness of the tail). There is 432for now no clear explanation for the physical process to which the UM parameter estimates 433differences could be attributed, and further investigations would be required to clarify this. 434 In order to be able to compare these results in time with those obtained on the vertical 435column in the previous section; one has to consider similar time scales. According to its size 436the duration taken by a drop to fall through the $36 \mathrm{~m}$ vertical column is between few seconds 437and few tens of seconds (observed drop velocity are between 0.5 and $10 \mathrm{~m} . \mathrm{s}^{-1}$ ). This range of 438scales corresponds to the middle regime for which there is no clear scaling behaviour. It might 439be due to the fact that the data is considered for the whole event and not only for the most 440intense portions as it is done for the spatial $1 \mathrm{D}$ analysis. In order to test this hypothesis a TM 441analysis was carried out considering only on the one minute long time series of the 09-244422012 event. Successive minutes starting every 15 s (moving window) were considered during 443the event and the scaling was quantified on the range $2 \mathrm{~s}$ to $33 \mathrm{~s}$ which would corresponds to 444the regime observed in the vertical column. It appears that some minutes exhibit good scaling 
445(with $r^{2}$ greater than 0.98) and other no (with $r^{2}$ smaller than 0.90 ) without any direct link with 446the corresponding rainfall intensities. This confirms the bad scaling observed on this range of 447scale with longer time series, and invalidates the tested hypothesis. It means that more 448investigations on the 3+1D structure of rainfall would be needed to properly link the results 449between the two types of analyses which are furthermore affected by the bias associated with 450the coarse ballistic assumption in this paper.

451

452

4535) Rain drop accumulation analysis

454

4555.1) 2D rain drop accumulation for a given number of consecutive drops

456

457 The aim of this section is to study the spatial distribution of the accumulation of a 458given number $\mathrm{N}$ of consecutive drops at ground level (more precisely at the height of the 4592DVD) over the sampling area of the device $\left(11 \times 11 \mathrm{~cm}^{2}\right)$. A 634 x 634 matrix corresponding 460to pixels seen by the 2DVD is created. To compute the pixel by pixel accumulation, the 461volume of a drop passing through the 2DVD is evenly distributed between all the pixels that 462are partially or totally obscured (information provided by the 2DVD), hence the unrealistic 463square shape of drops visible on the related figures. The drop accumulation is obtained by 464summing the contribution of $N$ consecutive drops to yield either a 2D rain accumulation map 465or a 2D drop occurrence map. Various $N$ (from 50 to 2000) were tested and yielded similar 466results. Finally a 512 x 512 pixels portion (a 9x9 $\mathrm{cm}^{2}$ area in the middle of the domain which 467enables to remove potential bias due to instrumental side effects) is extracted to carry out 468fractal and multifractal analysis. Figure 7 displays an example of a rain drop accumulation of 469150 consecutive drops during the 10-23-2013 event. As in section 3, each (natural) drop 
470accumulation map is compared with a homogenised version, i.e. a synthetic drop

471accumulation map. This synthetic map is obtained by keeping the same drops with their size 472and volume, but randomly homogeneously distributing their position over the sampling area 473with the help of a uniform law. A natural drop accumulation and one of its homogeneous 474versions are displayed in Fig. 7. Finally the duration needed to record $N$ consecutive drops is 475also retrieved and will be analysed.

476

4775.2) Fractal and multifractal analysis

478

479 The box-counting technique (i.e. Eq. 1 in log-log plot) was applied to drop occurrence 480accumulation maps to determine their fractal dimension. It is first applied to the drop centres, 481and then to the pixels occluded by drops. Both are displayed in Fig. 8.a and 8.b for the 10-234822013 event with 150 drops per maps respectively. The numbers of selected pixels $N(\lambda)$ were 483averaged over the ensemble of 1179 pictures recorded on a total duration of $12 \mathrm{~h}$ for each 484resolution $\lambda$ (Eq. 1). Very similar results are observed for the 4 other events and therefore not 485shown here. Fig. 8 displays a plateau for scales smaller than approximately $12 \mathrm{~mm}(\lambda \geq 8)$, due 486to the fact that the resolution of pixels is too high with respect to the number of drop centres. 487For larger scales ( 23 - $92 \mathrm{~mm}, 4 \geq \lambda \geq 1$ ), the box-counting technique yields a fractal dimension 4882, meaning that the drop centres are homogeneously distributed and filling the whole 489embedding space. The disagreement with the result of Lovejoy and Schertzer (1990) who 490recorded the position of 452 drops on $128 \mathrm{~cm}$ x $128 \mathrm{~cm}$ chemically treated blotting paper, 491discussed and finally confirmed by Gabbella et al. (2001) seems to be merely due to the fact 492that the scale of the sampling area is too small to reach one of the inhomogeneous regimes. 493This is in agreement with the fact that the rainfall field exhibited a scaling structure down to $4940.5 \mathrm{~m}$ and a homogeneous distribution for smaller scales (see Sec. 3) 
496centres, similar features are retrieved for large scales, but for small scales two fractal 497dimensions are obtained: respectively 1.1 in the range $23 \mathrm{~mm}-1 \mathrm{~mm}(4 \leq \lambda \leq 128$, red line in 498Fig. 8.b) and 1.65 for scales smaller than $0.7 \mathrm{~mm}$ (128 $\leq \lambda$, green line in Fig. 8.b). This 499disagreement with the homogeneity of the drop centres might be due to the variability of the 500rain volumes (due the drops’ sizes) that occurs over smaller scales.

501 A TM analysis was carried out on rain accumulation maps (not shown here). The main 502results are that the observed scaling is very bad, and that the curves obtained by analysing the 503random-position pictures are very similar. This is simply a confirmation that there is no clear 504scaling regime for scales smaller than $9 \mathrm{~cm}$ which as already mentioned was expected from 505the results of section 3 .

506

5075.3) Analysis of the distribution of duration needed for each picture 508

509 In this section, the durations needed to obtain $N$ consecutive drops through the 510sampling area are analysed. Figure 9.a displays the strong fluctuations of the series of theses 511durations for the 10-27-2013 event and $N=350$. The event lasted 9.3 hours with a total 512number of series of $N$ drops equal to 302. Figure 9.b displays the exceedance probability 513distribution of the durations in a log-log plot, which exhibits a power-law tail (linear slope in 514the log-log plot). This feature is opposed to Poisson statistics that yield exponential fall-off of 515the probability distributions. For this event the power-law is visible for scales ranging from 30 516s to $10 \mathrm{~min}$. Larger scales are not taken into account since they correspond to inter-event 517features rather than the intra-event ones which are accessible with the available data set. For a 518more extensive study on inter-event analysis refer to Lavergnat and Golé (1998) who also 519observed power-law behaviour in a slightly different context since they considered much 
520longer time series and $N=1$. The exponent of the power law is found roughly equal to $0.8-0.9$ 521for $N=350$ and similar values are found for other $N$ with a slight tendency to decrease with 522increasing $N$ ( $N$ ranging from 50 to 500, with steps of 50, were tested). The same power law 523behaviour is also observed for the 10-23-2012 event. For the 10-26-2013 and 09-24-2012 524events, the range of available durations does not enable to see the power law (especially true 525for the 09-24-2012 event). For the 10-20-2012, the range of available duration is similar to the 526one for the 10-27-2013 and 10-23-2013 events but the scaling is not very good as it was 527already noticed while analysing the $1 \mathrm{~ms}$ time series.

\section{6) Conclusion}

534 In this paper 2DVD data of 4 rainfall events were analysed to grasp some insights into 535the 3+1D structure of the rainfall field at the drop scale. Firstly, based on a ballistic 536assumption, vertical $36 \mathrm{~m}$ high rainfall columns above the measuring device are 537reconstructed. It appears that during the most intense portions of the events a good scaling 538behaviour is retrieved on scales ranging from $0.5 \mathrm{~m}$ to $36 \mathrm{~m}$. This inner scale seems to depend 539on the drop size distribution and larger scale data would be needed to investigate more in 540depth this dependency. These observations are incompatible with a homogeneous distribution 541of drops. On the other hand for smaller scales the observations are in agreement with the 542hypothesis of a homogeneous distribution of drops. Results show that integrated values (such 543as number of drops or LWC) are well represented by UM simulations for heaviest rainfall 544period on scales ranging from $0.5 \mathrm{~m}$ to $36 \mathrm{~m}$. An interesting future perspective, that would 
545require more investigations which are beyond the scope of this paper, would be to actually 546generate a rainfall simulator at drop scale which enables to reproduce observations. This 547simulator would need to be capable of integrating break-up and coalescence as well as small 548scale turbulence wind effect in order to overcome the limitations of the ballistic assumption 549used here. Results show that using a multifractal framework would be relevant, to design this 550rainfall simulator.

551 Secondly, 1 ms rain rate time series are analysed. With such resolution it is possible to 552actually grasp the underlying assumption of the multifractal framework that rainfall is 553extremely concentrated on small portions of time or space. The mathematical interpretation is 554that rain accumulation is actually a measure that is singular with respect to the usual space / 555time measures (Lebesgue measures). Two scaling regimes are confirmed with a transition in 556between. The first one for very small scales ( $1 \mathrm{~ms}$ - few tens of ms) is actually fractal and 557corresponds to rather individual drops. The large scale multifractal regime (few min, few tens 558of min) correspond to a kind of collective regime of drops and UM parameter values depend 559on the event. We found that multifractality index $\alpha$ ranges between 1 and 2 and the 560codimension of the mean intermittency $C_{1}$ between 0.2 and 0.5 .

561 Thirdly, $N$ consecutive drop accumulation maps observed by the measuring device are 562analysed. Fractal spatial analyses were carried out on the actual drop accumulation, as well as 563homogeneised versions. Both yield very similar results and do not exhibit scaling features. 564This is due to the fact that the size of the sampling area $(11 \mathrm{~cm} \mathrm{x} 11 \mathrm{~cm})$ is smaller than the 565inner scale of the scaling regime identified with the analysis of the vertical columns. 566Nevertheless, the distribution of the durations needed to record $N$ drops, exhibits a power law 567tail, which invalidates the usual hypothesis of a Poisson distribution

568 The spatio-temporal structure of the rainfall field is investigated through the analysis 569of $1 \mathrm{D}$ (vertical column, time series) or 2D (drop accumulations cuts of the spatio-temporal 
570field. Further investigations would be needed to establish a rigorous link between the full 571underlying process and these cuts. This would enable to improve our representation of the full $5723+1 \mathrm{D}$ structure of the field with all its drops which is the ultimate goal. We showed that 573during heaviest rainfall period the commonly used Poisson hypothesis is not valid and results 574suggest that a framework relying on multifractals may help overcome some of current 575discrepancies. Finally it should be mentioned that this work has some strong consequences on 576the remote sensing of rainfall with weather radar for instance. Indeed radar rainfall retrieval 577algorithms usually assume that drops are homogeneously distributed within the scanned 578volume which is not necessarily the case especially during the most intense portions of 579rainfall events. This issue of non-uniform beam filling issue is experienced by radar analysts. 580Given the scale gap between the volume scanned by radar and the studied volume of the 581vertical column (at least a ratio of $10^{6}$ between the volumes), it is difficult to be more 582affirmative, but it converges with studies of the speckle effect, due to coherent backscattering 583of inhomogeneously distributed drops, which underpinned strong biases in the rainfall rate 584estimates. Some theoretical investigations of this effect with the help of multifractals have 585already been carried out (Lovejoy et al. 1996, Schertzer et al. 2012), and further empirical 586analysis with the reconstructed columns could help to better quantify the actual consequences 587of this effect. The variability observed between consecutive snapshots of vertical columns 588(done every second in this paper) also suggests that the sampling uncertainty of radar data due 589to limited revisit time in the scanning strategies should be investigated more in depth. These 590two issues will be analysed in future work.

591

592

593Acknowledgments 
594The authors greatly acknowledge partial financial support form the Chair "Hydrology for 595Resilient Cities” (sponsored by Veolia) of Ecole des Ponts ParisTech, EU NEW-INTERREG 596IV RainGain Project (www.raingain.eu), and EU Climate KIC Blue Green Dream project 597(www.bgd.org.uk). Tim Raupach is also acknowledged for having selected the studied events 598and providing Fig. 1.

599

600

601References

602

603Anselmet, F., Antonia, R.A., Danaila, L., 2001: Turbulent flows and intermittency in 604 laboratory experiments. Planet. Space Sci., 49, 1177-1191.

605Battaglia, A., Rustemeier, E., Tokay, A., Blahak, U. and Simmer, C., 2010: PARSIVEL Snow 606 Observations: A Critical Assessment. J. Atmos. Oceanic Technol., 27(2), 333-344.

607Cao, Q. et al., 2008: Analysis of Video Disdrometer and Polarimetric Radar Data to

608 Characterize Rain Microphysics in Oklahoma. J. Appl. Meteor. Climatol., 47(8), 22386092255.

610Cotton, W.R. and Gokhale, N.R., 1967: Collision, coalescence, and breakup of large water 611 drops in a vertical wind tunnel. J. Geophys. Res., 72 (16), 4041-4049.

612de Lima, M.I.P. and de Lima, J., 2009: Investigating the multifractality of point precipitation 613 in the Madeira archipelago. Nonlin. Processes Geophys., 16(2), 299-311.

614de Lima, M.I.P. and Grasman, J., 1999: Multifractal analysis of 15-min and daily rainfall from 615 a semi-arid region in Portugal. J. Hydrol., 220(1-2), 1-11.

616de Montera, L., Barthes, L., Mallet, C. and Gole, P., 2009: The Effect of Rain-No Rain 617 Intermittency on the Estimation of the Universal Multifractals Model Parameters. $J$. 618 Hydrometeor., 10(2), 493-506. 
619Desaulnier-Soucy, N., Lovejoy, S. and Schertzer, D., 2001: The continuum limit in rain and 620 the HYDROP experiment. J. Atm. Res., 59-60, 163-197.

621Ducrocq et al., 2014: HyMeX-SOP1, the field campaign dedicated to heavy precipitation and 622 flash flooding in the northwestern Mediterranean. Bull. Amer. Meteor. Soc., doi: $623 \quad$ http://dx.doi.org/10.1175/BAMS-D-12-00244.1

624Fitton, G., Tchiguirinskaia, I., Schertzer, D., Lovejoy, S., 2011: Scaling Of Turbulence In The 625 Atmospheric Surface-Layer: Which Anisotropy?. Journal of Physics: Conference $626 \quad$ Series, 318, doi:10.1088/1742-6596/318/7/072008

627Fraedrich, K. and Larnder, C., 1993: Scaling regimes of composite rainfall time-series. Tellus 628 Series a-Dynamic Meteorology and Oceanography, 45A(4), 289-298.

629Frasson, R. P. d. M., L. K. d. Cunha, and W. F. Krajewski (2011), Assessment of the Thies 630 optical disdrometer performance, Atmos. Res., 101, 237-255, doi:

$631 \quad$ 10.1016/j.atmosres.2011.02.014.

632Gabella, M., Pavone, S., Perona, G., 2001: Errors in the estimate of the fractal correlation 633 dimension of raindrop spatial distribution. J. Appl. Meteor., 40, 664-668.

634Gires, A. et al., 2012: Quantifying the impact of small scale unmeasured rainfall variability on 635 urban runoff through multifractal downscaling: A case study. J. Hydrol., 442-443, 117$636 \quad 128$.

637Gires, A., Tchiguirinskaia, I., Schertzer, D. and Lovejoy, S., 2011: Analyses multifractales et 638 spatio-temporelles des precipitations du modele Meso-NH et des donnees radar.

$639 \quad$ Hydrol. Sci. J., 56(3), 380-396.

640Gires, A., Tchiguirinskaia, I., Schertzer, D. and Lovejoy, S., 2013: Development and analysis 641 of a simple model to represent the zero rainfall in a universal multifractal framework. $642 \quad$ Nonlin. Processes Geophys., 20(3), 343-356. 
643Gires, A. et al., 2014: Influence of small scale rainfall variability on standard comparison 644 tools between radar and rain gauge data. Atmos. Res., 138(0), 125-138.

645Hubert, P. et al., 1993: Multifractals and extreme rainfall events. Geophys. Res. Lett., 20, 931646934.

647Jaffrain, J. and Berne, A., 2011. Experimental Quantification of the Sampling Uncertainty 648 Associated with Measurements from PARSIVEL Disdrometers. J. Hydrometeor., 649 12(3): 352-370.

650Jameson, A.R. and Kostinski, A.B., 1998: Fluctuation Properties of Precipitation. Part II:

651 Reconsideration of the Meaning and Measurement of Raindrop Size Distributions. J. $652 \quad$ Atmos. Sci., 55(2), 283-294.

653Jameson, A.R., Kostinski, A.B. and Kruger, A., 1999: Fluctuation Properties of Precipitation. 654 Part IV: Finescale Clustering of Drops in Variable Rain. J. Atmos. Sci., 56(1), 82-91. 655Joss, J. and Waldvogel, A., 1967: Ein spektrograph fur nieder chlagstropfen mit automatischer 656 auswertung (A spectrograph for raindrops with automatic interpretation). Pure Appl. 657 Geophys. Rev. A., 68, 240-246.

658Kostinski, A.B. and Jameson, A.R., 1997: Fluctuation Properties of Precipitation. Part I: On 659 Deviations of Single-Size Drop Counts from the Poisson Distribution. J. Atmos. Sci., $660 \quad$ 54(17), 2174-2186.

661Krajewski, W.F. et al., 2006: DEVEX-disdrometer evaluation experiment: Basic results and 662 implications for hydrologic studies. Adv. Wat. Resour., 29(2), 311-325.

663Kruger, A. and Krajewski, W.F., 2002: Two-Dimensional Video Disdrometer: A Description. $664 \quad$ J. Atmos. Oceanic Technol., 19(5), 602-617.

665Ladoy, P., Schmitt, F., Schertzer, D. and Lovejoy, S., 1993: The multifractal temporal 666 variability of Nimes rainfall data. Comptes Rendus de l'Academie Des Sciences Serie $667 \quad$ Ii, 317(6), 775-782. 
668Lavergnat, J. and Golé, P., 1998: A Stochastic Raindrop Time Distribution Model. J. Appl. 669 Meteor., 37(8), 805-818.

670Lazarev, A., Schertzer, D., Lovejoy, S. and Chigirinskaya, Y., 1994: Unified multifractal 671 atmospheric dynamics tested in the tropics: part II, vertical scaling and Generalized 672 Scale Invariance. Nonlin. Processes Geophys., 1, 115-123.

673Lilley, M., Lovejoy, S., Desaulniers-Soucy, N. and Schertzer, D., 2006: Multifractal large 674 number of drops limit in rain. J. Hydrol., 328(1-2), 20-37.

675Lovejoy, S., Duncan, M.R. and Schertzer, D., 1996: Scalar multifractal radar observer's 676 problem. J. Geophys. Res., 101, 26479-26492.

677Lovejoy, S. and Schertzer, D., 1990: Fractals, raindrops and resolution dependence of rain 678 measurements. J. Appl. Meteor., 29(11), 1167-1170.

679Lovejoy, S. and Schertzer, D., 1995: Multifractals and rain. New Uncertainty Concepts in 680 Hydrology and Hydrological modelling, A.W. Kundzewicz, Ed., Cambridge Press, 62$681 \quad 103$.

682Lovejoy, S. and Schertzer, D., 2008: Turbulence, rain drops and the $\mathrm{l}^{* *} 1 / 2$ number density 683 law. New J. of Physics, 10, 32pp.

684Lovejoy, S., Schertzer, D. and Allaire, V., 2008: The remarkable wide range spatial scaling of 685 TRMM precipitation. J. Atmos. Research, 90, 10-32.

686Mandapaka, P.V., Lewandowski, P., Eichinger, W.E. and Krajewski, W.F., 2009: Multiscaling 687 analysis of high resolution space-time lidar-rainfall. Nonlin. Processes Geophys., $688 \quad$ 16(5), 579-586.

689Nykanen, D.K. and Harris, D., 2003: Orographic influences on the multiscale statistical 690 properties of precipitation. J. Geophys. Res.-Atm., 108(D8),

691Olsson, J., 1995: Limits and characteristics of the multifractal behavior of a high-resolution 692 rainfall time series. Nonlin. Processes Geophys., 2, 23-29. 
693Royer, J.-F., Biaou, A., Chauvin, F., Schertzer, D. and Lovejoy, S., 2008: Multifractal analysis

694 of the evolution of simulated precipitation over France in a climate scenario. C.R.

695 Geoscience, 340, 431-440.

696Salles, C., Creutin, J.D. and Sempere-Torres, D., 1998: The optical spectropluviometer 697 revisited. J. Atmos. Oceanic Technol., 15(5), 1215-1222.

698Schertzer, D. and Lovejoy, S., 1987: Physical modelling and analysis of rain and clouds by 699 anisotropic scaling and multiplicative processes. J. Geophys. Res., 92(D8), 9693-9714. 700Schertzer, D. and Lovejoy, S., 1997: Universal multifractals do exist!: Comments. J. Appl. $701 \quad$ Meteor., 36(9), 1296-1303.

702Schertzer D, Tchiguirinskaia I, Lovejoy S, Hubert P., 2010: No monsters, no miracles: in 703 nonlinear sciences hydrology is not an outlier! Hydrol. Sci. J., 55(6), 965-979.

704Schertzer, D. and Lovejoy, S., 2011: Multifractals, generalized scale invariance and 705 complexity in geophysics. International Journal of Bifurcation and Chaos, 21(12), $706 \quad 3417-3456$.

707Schertzer, D., Tchiguirinskaia, I. and Lovejoy, S., 2012: Getting higher resolution rainfall 708 estimates: X-band radar technology and multifractal drop distribution. Proceedings of 709 the Weather Radar and Hydrology symposium held in Exeter, UK, April 2011, IAHS $710 \quad$ Publ. 351.

711Tchiguirinskaia, I., Salles, C., Hubert, P., Schertzer, D., Lovejoy, S., Creutin, J. D. \& 712 Bendjoudi, H., 2003: Mulitfractal analysis of the OSP measured rain rates over time 713 scales from millisecond to day. In: IUGG2003, Sapporo

714Tessier, Y., Lovejoy, S., Hubert, P., Schertzer, D. and Pecknold, S., 1996: Multifractal analysis 715 and modeling of rainfall and river flows and scaling, causal transfer functions. $J$.

716 Geophys. Res.-Atm., 101(D21), 26427-26440. 
717Tessier, Y., Lovejoy, S. and Schertzer, D., 1993: Universal Multifractals: theory and

718 observations for rain and clouds. J. Appl. Meteor., 32(2), 223-250.

719Thurai, M. and Bringi, V.N., 2005: Drop Axis Ratios from a 2D Video Disdrometer. J. Atmos. $720 \quad$ Oceanic Technol., 22(7), 966-978.

721Thurai, M., Peterson, W.A., Tokay, A., Schutz, C. and Gatlin, P., 2011: Drop size distribution 722 comparisons between Parsivel and 2-D video disdrometers. Adv. Geosci., 30, 3-9.

723Tokay, A., Petersen, W.A., Gatlin, P. and Wingo, M., 2013: Comparison of Raindrop Size 724 Distribution Measurements by Collocated Disdrometers. . J. Atmos. Oceanic Technol., $725 \quad 30(8), 1672-1690$

726Uijlenhoet, R., Stricker, J.N.M., Torfs, P.J.J.F. and Creutin, J.D., 1999: Towards a stochastic 727 model of rainfall for radar hydrology: testing the Poisson homogeneity hypothesis.

728 Physics and Chemistry of the Earth, Part B: Hydrology, Oceans and Atmosphere, $729 \quad$ 24(6), 747-755.

730Verrier, S., de Montera, L., Barthes, L. and Mallet, C., 2010: Multifractal analysis of African 731monsoon rain fields, taking into account the zero rain-rate problem. J. Hydrol., 389(1-2), 111732120.

733

734

735Figure caption list

736

737Figure 1: Picture of the installed 2DVD in Le Pradel, Ardèche, France 738

739Figure 2: (a) Example of a reconstruction for a $26 \mathrm{~cm}$ vertical column; dimensions are in mm, 740drops have been coloured according to size and their diameter has been multiplied by 4 to 741improve visibility (b) Illustration of the $36 \mathrm{~m} \mathrm{x} 11 \mathrm{~cm}$ x $11 \mathrm{~cm}$ reconstructed column divided 
742into smaller boxes. (c) Vertical evolution of the LWC (in g. $\mathrm{m}^{-3}$ ) within the vertical column for 743the reconstructed field (top in black) and simulated assuming homogenous distribution of 744drop positions (bottom in red) for an instant of the 09-24-2012 event. The horizontal axis 745corresponds to the box number (0: ground level, 8192: top of the reconstructed column). 746

747Figure 3: (a) Spectral analysis of the vertical column snapshots for 60 seconds starting on 24 748September 2012 02:17 UTC. (b) TM analysis of the same data (points) and the corresponding 749synthetic field-same drops (size and velocity) but their position is randomly (uniformly) 750assigned (solid, curved lines). The linear regressions (straight lines on the right side of the 751figure) are performed only for large scales, i.e. 0.5 - $36 \mathrm{~m}$ (c) Same as in (b) zoomed on the 752large scales (d) Spectral analysis of the corresponding synthetic field.

\section{3}

754Figure 4: Illustration of the absence of scaling in columns with too low number of drops. (a) 755Same as in Fig. 3.b but for 60 time steps starting at 02:23UTC. (b) Scatter plot of $r^{2}$ for $q=1.5$ 756in the TM analysis vs. the average number of drops in the studied columns. (c) Scatter plot of 757the average number of drops in the studied columns vs. the indicative rain rate computed at 758ground level. 759

760Figure 5: Figures are plotted for the 09-24-2012 event (a) Temporal evolution of the number 761of drops passing through the sampling area with 1ms time steps. (b), (c) and (d) Temporal 762evolution of the rain rate during the same event with time steps of $1 \mathrm{~ms}$, $1 \mathrm{~s}$ and $1 \mathrm{~min}$ 763respectively. (e) Temporal evolution of the average mass weighted diameter. (f), (g) and (h): 764same as in (b), (c) and (d) expressed in $\log _{\lambda}$. 
766Figure 6: Spectral analysis (a) and TM analysis (b) data corresponding to 140 min of the 1076723-2013 event with 1 ms time steps (time series of length $2^{23}$ ).

768

769Figure 7: Top: natural drop accumulation: rain accumulation map (left) and occurrence map 770(right) for 150 consecutive drops during the 10-23-2013 event. Bottom: Same as Top but for a 771homogenised version of the drop accumulation (see text for details). The colour scale is in $772 \mathrm{~mm} \times 10^{-3}$

773

774Figure 8: Fractal analysis (Eq. 1 in log-log plot) of the drop position for 10-23-2013 event 775with 150 drops per drop accumulation map (ensemble analysis on the 1179 pictures recorded 776on a total duration of 12h). (a) On the drop centres. (b) Considering all the pixels occluded by 777drops.

778

779Figure 9: (a) Duration need to record pictures with 350 drops during the 10-27-2013 event (b) 780The exceedance probability of the distribution of the durations in a log-log plot ( $\Delta \mathrm{t}$ and $\mathrm{x}$ are 781in s).

782

783

784

785

786

787

788

789

790 


\section{Tables}

795

\begin{tabular}{|l|l|l|l|}
\hline Event & $\begin{array}{l}\text { Approx } \\
\text { duration }(\mathrm{h})\end{array}$ & $\begin{array}{l}\text { Total depth } \\
(\mathrm{mm})\end{array}$ & Maximum rain \\
& 0.6 & 10.9 & rate $(\mathrm{mm} / \mathrm{h})$ \\
\hline $09-24-2012$ & 20 & 20.4 & 103 \\
\hline $10-26-2012$ & 8 & 21.4 & 45.9 \\
\hline $10-23-2013$ & 6 & 10.6 & 76.1 \\
\hline $10-27-2013$ & 6 & 76.9 \\
\hline
\end{tabular}

796Table 1: Basic features of the studied events

797

798

\begin{tabular}{|l|l|l|l|l|}
\hline Event & Studied & Large scale & $\alpha$ & $C_{1}$ \\
& duration (min) & range (min) & & \\
\hline $09-24-2012$ & 35 & $35-2$ & 1.95 & 0.21 \\
\hline $10-26-2012$ & 140 & $140-9$ & 1.7 & 0.3 \\
\hline $10-23-2013$ & 140 & $140-2$ & 1.3 & 0.3 \\
\hline $10-27-2013$ & 140 & $140-2$ & 1.03 & 0.47 \\
\hline
\end{tabular}

799Table 2: UM parameter estimates for large scales in the temporal analysis of $1 \mathrm{~ms}$ resolution

800time series for the 5 studied events.

801

802

803

804Figure list:

805 


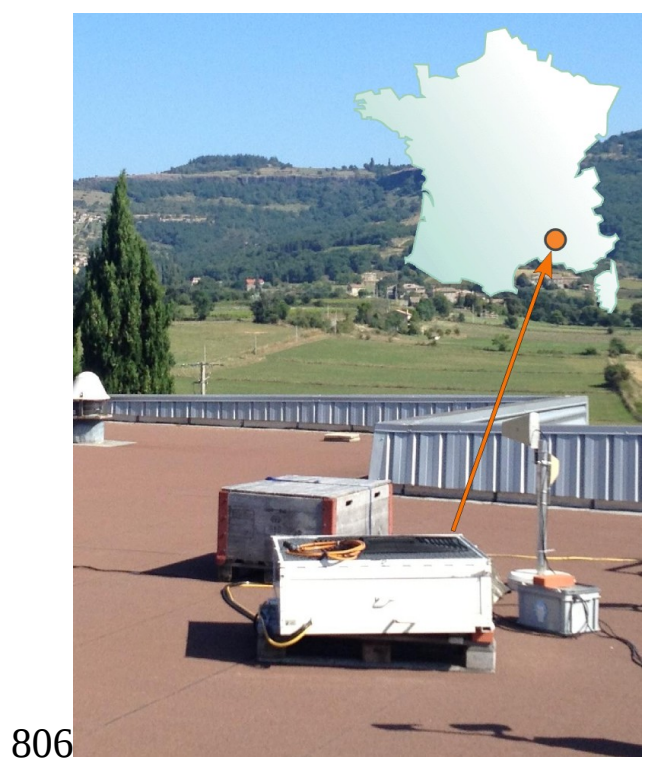

807Figure 1: Picture of the installed 2DVD in Le Pradel, Ardèche, France

808

(a)

2012-09-24 02:17:00.256000

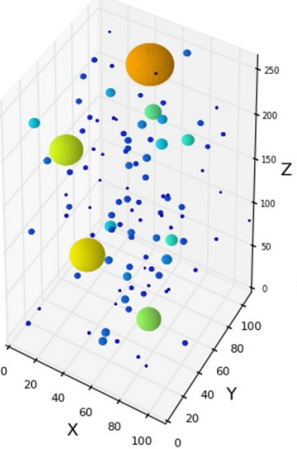

(b)

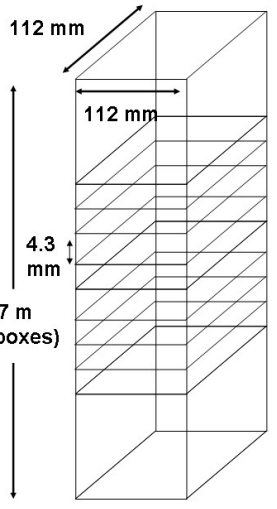

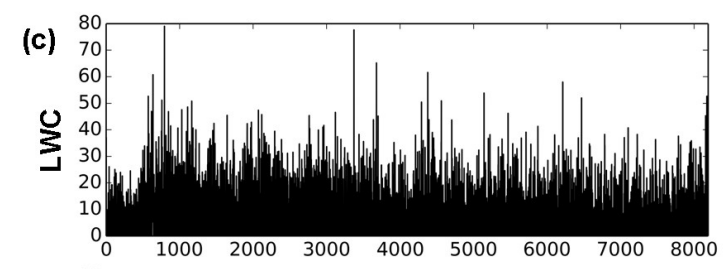

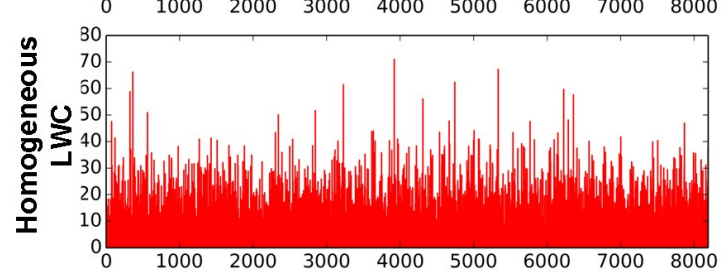

809

810Figure 2: (a) Example of a reconstruction for a $26 \mathrm{~cm}$ vertical column; dimensions are in mm,

811drops have been coloured according to size and their diameter has been multiplied by 4 to

812improve visibility (b) Illustration of the $36 \mathrm{~m} \mathrm{x} 11 \mathrm{~cm}$ x $11 \mathrm{~cm}$ reconstructed column divided

813into smaller boxes. (c) Vertical evolution of the LWC (in $\mathrm{g}^{\mathrm{m}} \mathrm{m}^{-3}$ ) within the vertical column for 814the reconstructed field (top in black) and simulated assuming homogenous distribution of 815drop positions (bottom in red) for an instant of the 09-24-2012 event. The horizontal axis 816corresponds to the box number (0: ground level, 8192: top of the reconstructed column). 

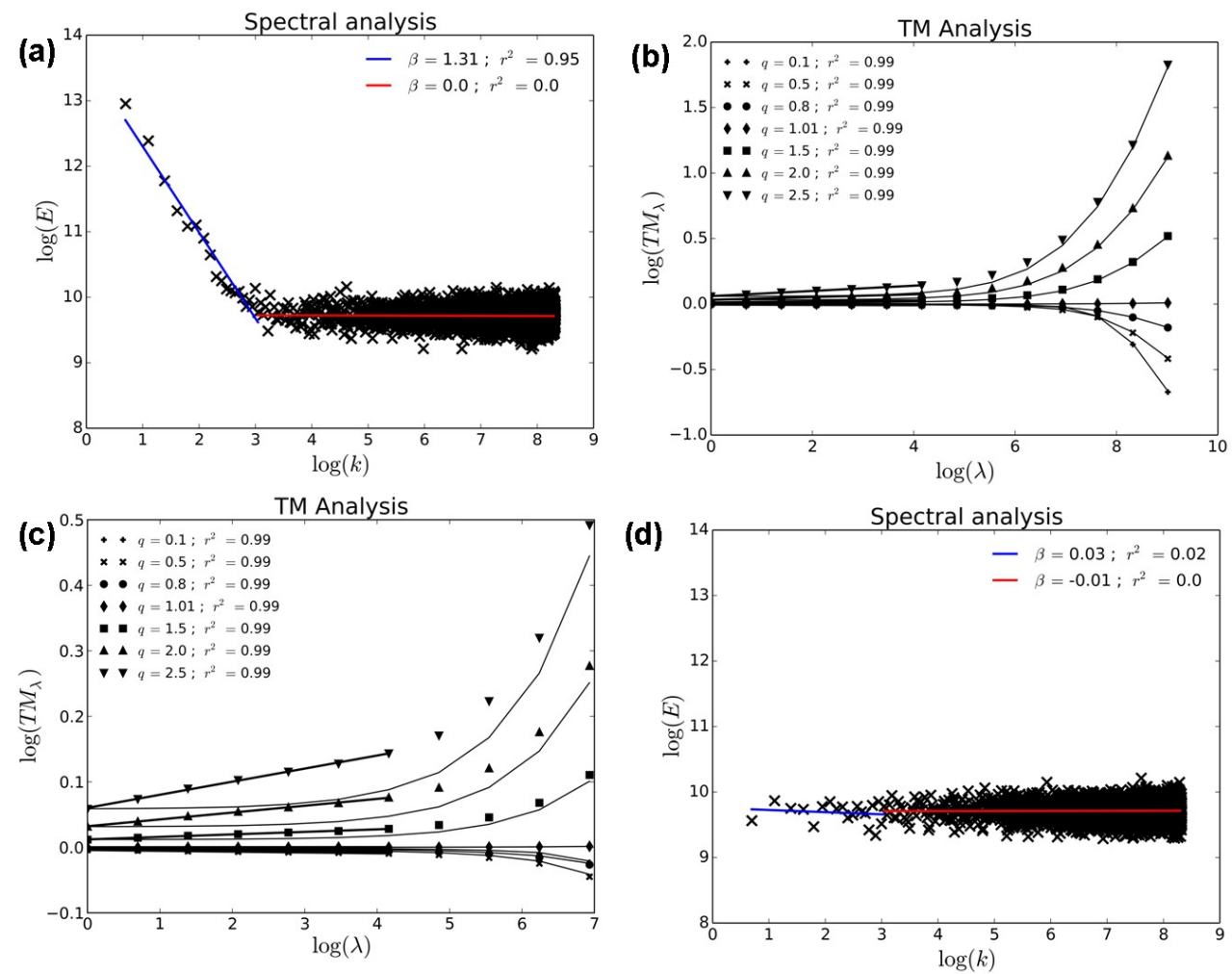

819

820Figure 3: (a) Spectral analysis of the vertical column snapshots for 60 seconds starting on 24 821September 2012 02:17 UTC. (b) TM analysis of the same data (points) and the corresponding 822synthetic field-same drops (size and velocity) but their position is randomly (uniformly) 823assigned (solid, curved lines). The linear regressions (straight lines on the right side of the 824figure) are performed only for large scales, i.e. 0.5 - 36 m (c) Same as in (b) zoomed on the 825large scales (d) Spectral analysis of the corresponding synthetic field.
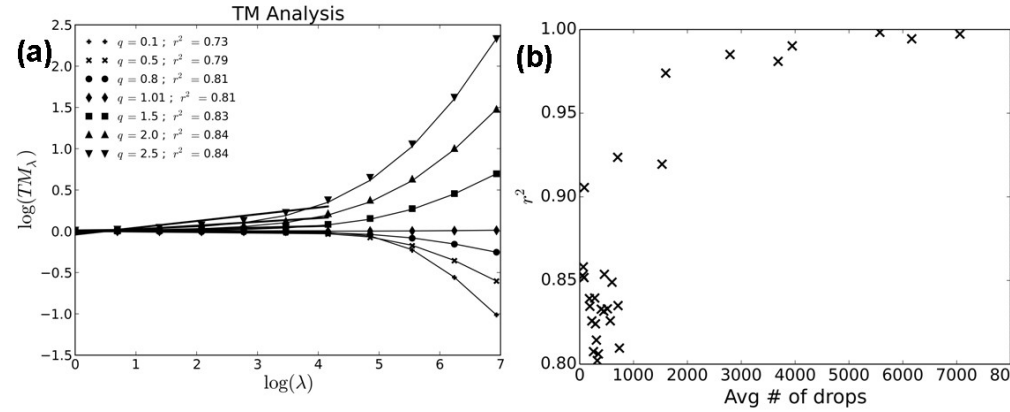
Avg \# of drops

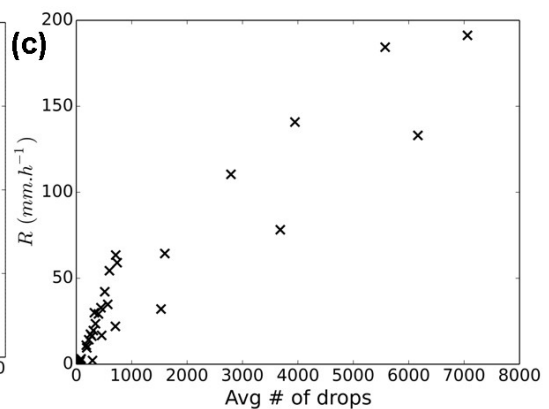

Avg \# of drops 
830Figure 4: Illustration of the absence of scaling in columns with too low number of drops. (a) 831Same as in Fig. 3.b but for 60 time steps starting at 02:23UTC. (b) Scatter plot of $r^{2}$ for $q=1.5$ 832in the TM analysis vs. the average number of drops in the studied columns. (c) Scatter plot of 833the average number of drops in the studied columns vs. the indicative rain rate computed at 834ground level.

(a)

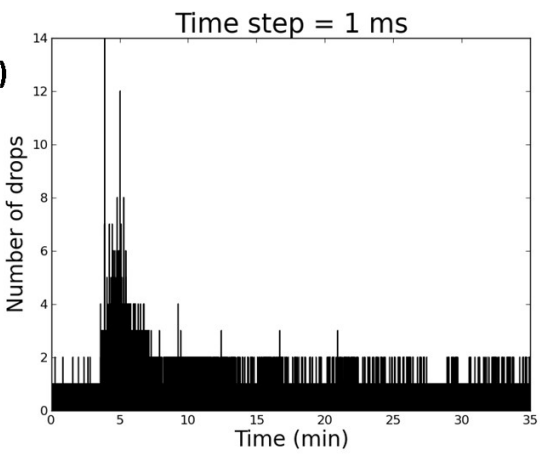

(b)

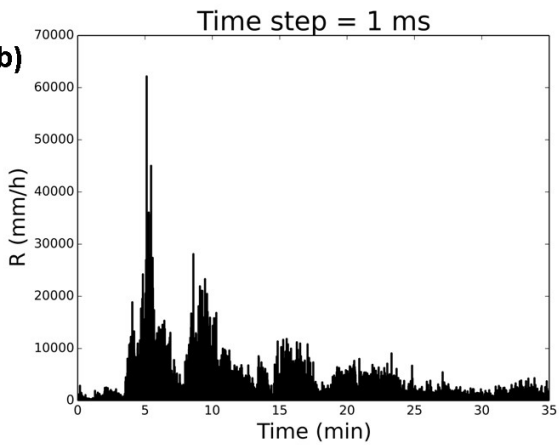

(c)

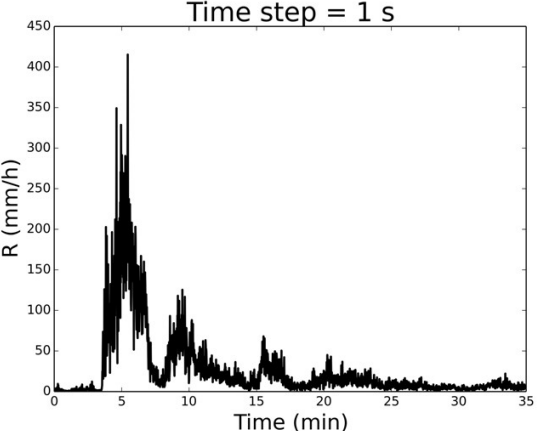

Time step $=1 \mathrm{~min}$

(d)

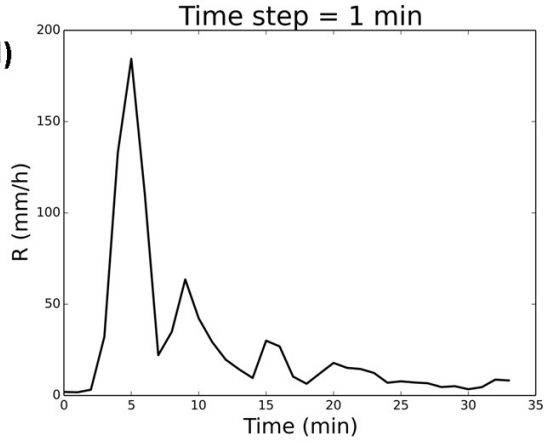

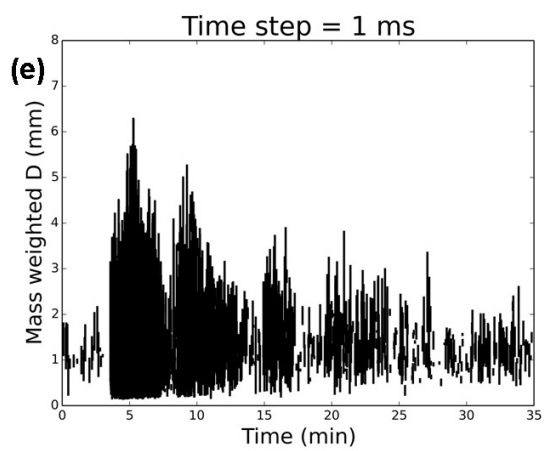
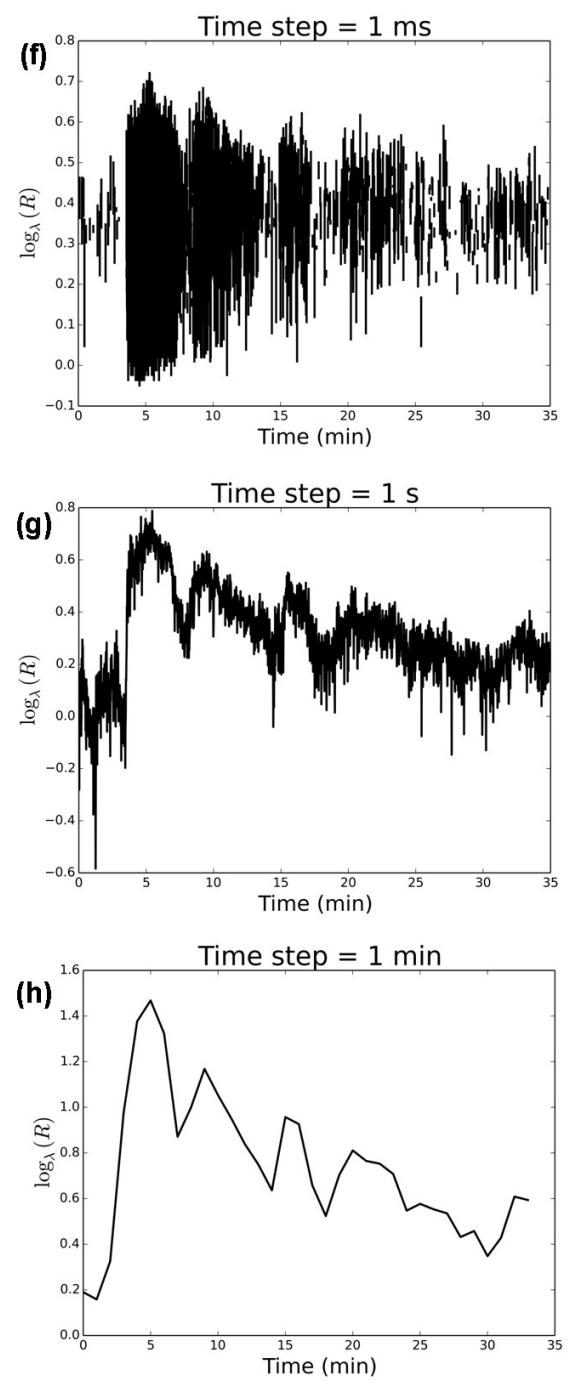
838Figure 5: Figures are plotted for the 09-24-2012 event (a) Temporal evolution of the number 839of drops passing through the sampling area with 1ms time steps. (b), (c) and (d) Temporal 840 evolution of the rain rate during the same event with time steps of $1 \mathrm{~ms}, 1 \mathrm{~s}$ and $1 \mathrm{~min}$ 841respectively. (e) Temporal evolution of the average mass weighted diameter. (f), (g) and (h): 842same as in (b), (c) and (d) expressed in $\log _{\lambda}$.

843
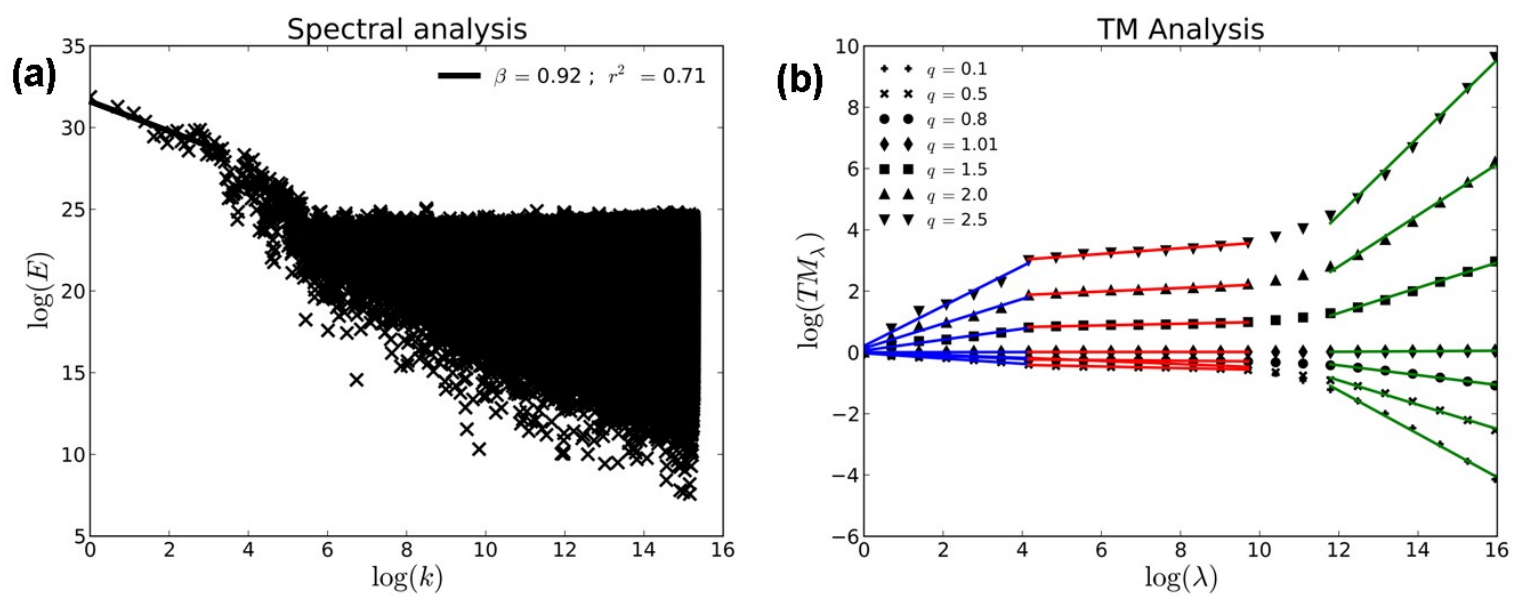

844

845Figure 6: Spectral analysis (a) and TM analysis (b) data corresponding to 140 min of the 1084623-2013 event with 1 ms time steps (time series of length $2^{23}$ ).

847

848 

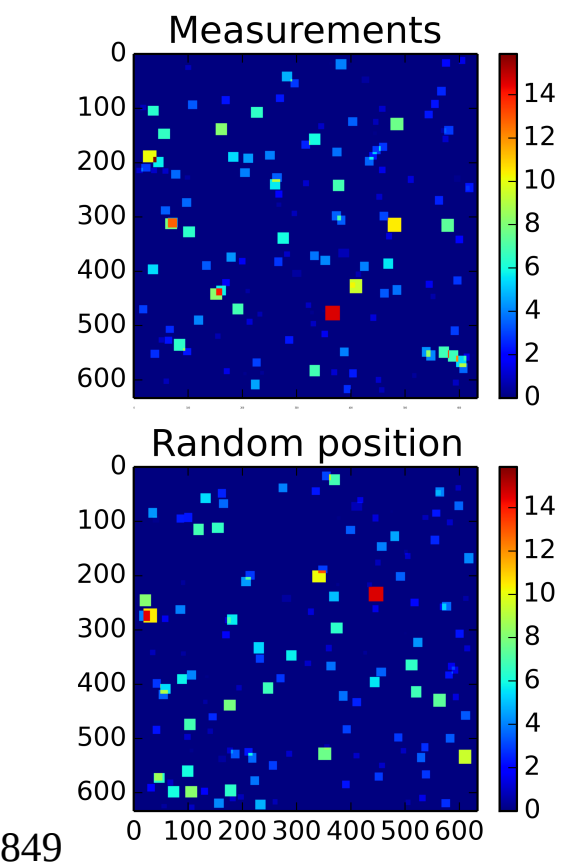

Measurements, occurence

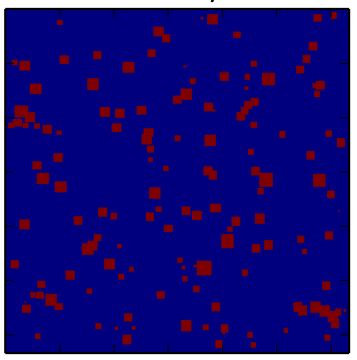

Random position, occurence

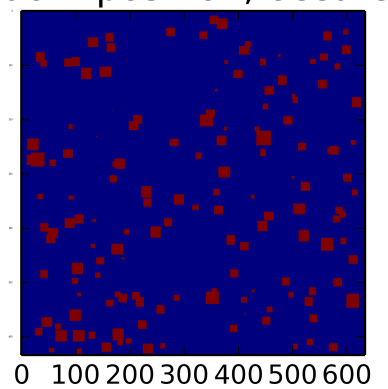

850Figure 7: Top: natural drop accumulation: rain accumulation map (left) and occurrence map 851(right) for 150 consecutive drops during the 10-23-2013 event. Bottom: Same as Top but for a 852homogenised version of the drop accumulation (see text for details). The colour scale is in $853 \mathrm{~mm} \times 10^{-3}$

854
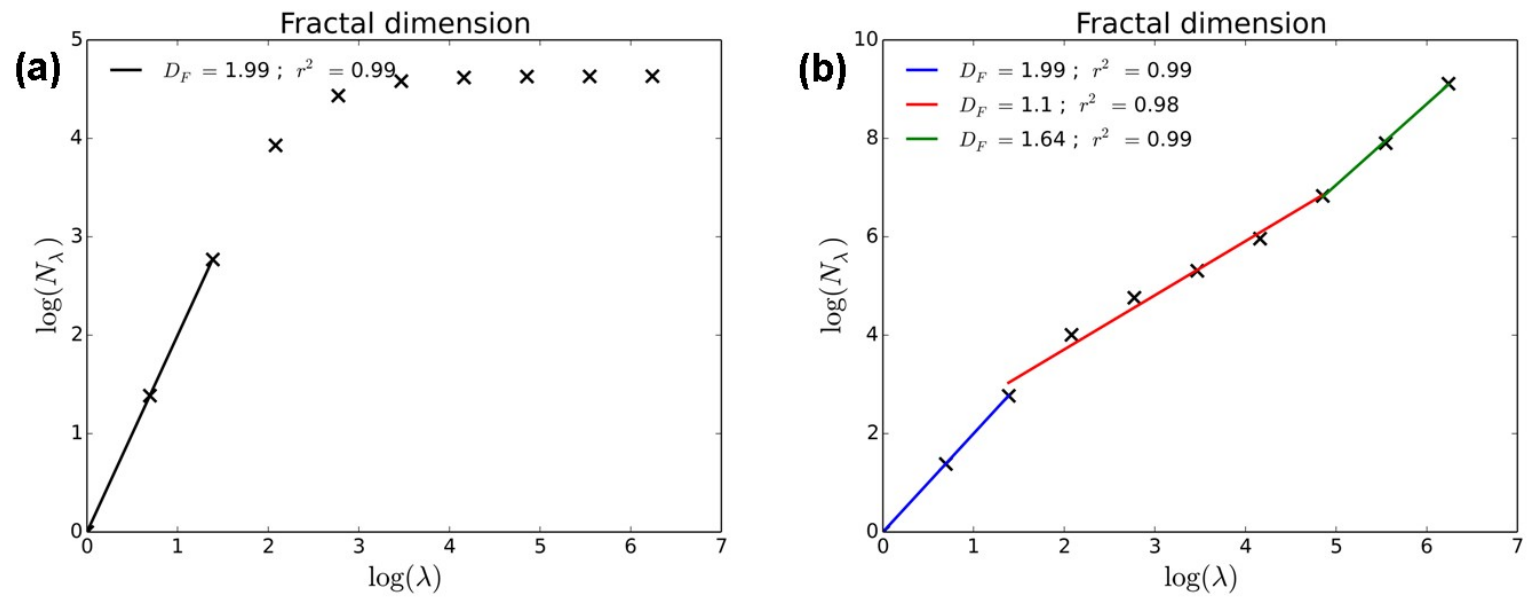

855

856Figure 8: Fractal analysis (Eq. 1 in log-log plot) of the drop position for 10-23-2013 event 857with 150 drops per drop accumulation map (ensemble analysis on the 1179 pictures recorded 858on a total duration of $12 \mathrm{~h}$ ). (a) On the drop centres. (b) Considering all the pixels occluded by 859drops. 
861
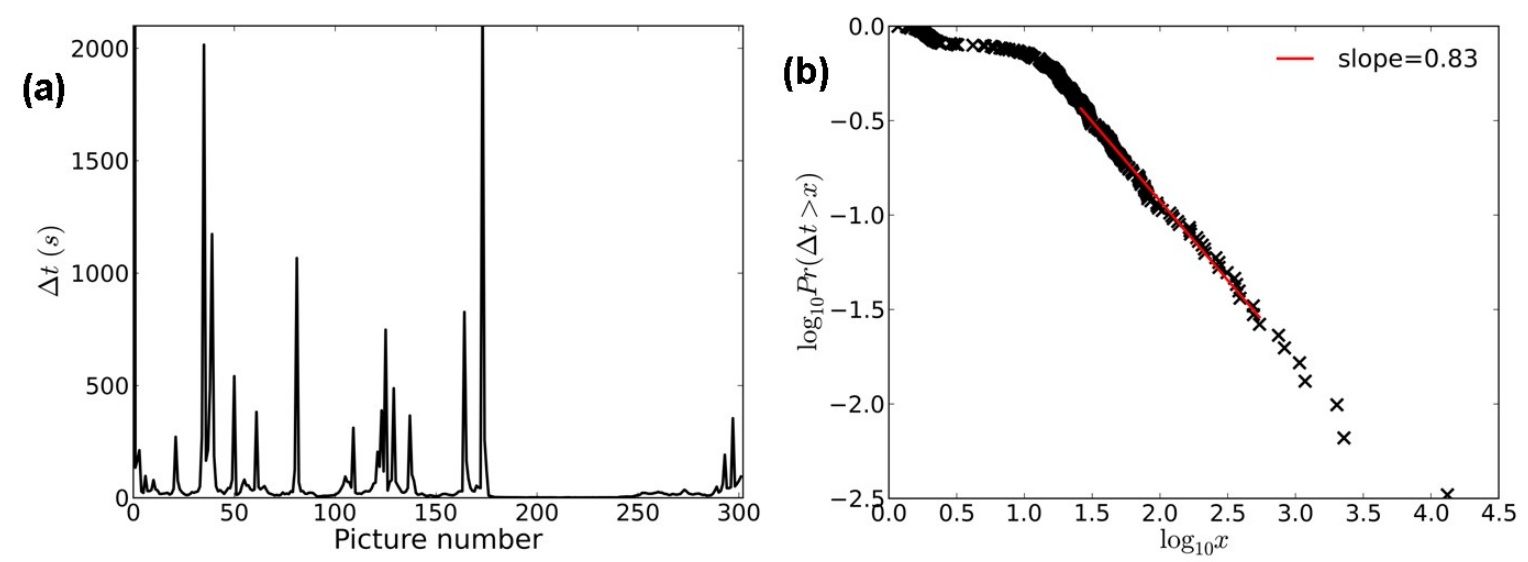

862

863Figure 9: (a) Duration need to record pictures with 350 drops during the 10-27-2013 event (b) 864The exceedance probability of the distribution of the durations in a log-log plot $(\Delta \mathrm{t}$ and $\mathrm{x}$ are 865in s).

866 